FACTA UNIVERSITATIS

Series: Mechanical Engineering Vol. 15, No 3, 2017, pp. 457 - 465

https://doi.org/10.22190/FUME170927025L

Original scientific paper

\title{
NUMERICAL COMPUTATION AND PREDICTION OF ELECTRICITY CONSUMPTION IN TOBACCO INDUSTRY
}

\author{
UDC 519.6
}

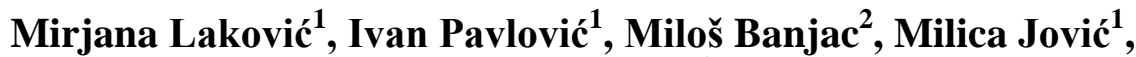 \\ Marko Mančić ${ }^{1}$
}

${ }^{1}$ Faculty of Mechanical Engineering, University of Niš, Serbia

${ }^{2}$ Faculty of Mechanical Engineering, University of Belgrade, Serbia

\begin{abstract}
Electricity is a key energy source in each country and an important condition for economic development. It is necessary to use modern methods and tools to predict energy consumption for different types of systems and weather conditions. In every industrial plant, electricity consumption presents one of the greatest operating costs. Monitoring and forecasting of this parameter provide the opportunity to rationalize the use of electricity and thus significantly reduce the costs. The paper proposes the prediction of energy consumption by a new time-series model. This involves time series models using a set of previously collected data to predict the future load. The most commonly used linear time series models are the AR (Autoregressive Model), MA (Moving Average) and ARMA (Autoregressive Moving Average Model). The AR model is used in this paper. Using the AR (Autoregressive Model) model, the Monte Carlo simulation method is utilized for predicting and analyzing the energy consumption change in the considered tobacco industrial plant. One of the main parts of the AR model is a seasonal pattern that takes into account the climatic conditions for a given geographical area. This part of the model was delineated by the Fourier transform and was used with the aim of avoiding the model complexity. As an example, the numerical results were performed for tobacco production in one industrial plant. A probabilistic range of input values is used to determine the future probabilistic level of energy consumption.
\end{abstract}

Key Words: Electricity Consumption, Forecasting of Electricity, AR Model, Monte Carlo Simulation, Seasonality Pattern

Received September 27, 2017 / Accepted November 18, 2017

Corresponding author: Ivan Pavlović

Faculty of Mechanical Engineering, University of Niš, A. Medvedeva 14, 18000 Niš, Serbia

E-mail: pivan@masfak.ni.ac.rs 


\section{INTRODUCTION}

Following the deregulations that took place in many countries over the last few decades, energy markets have been rapidly evolving and bringing to the attention of practitioners and researchers the challenging problems from the modeling perspective. Energy consumption has been increasing constantly due to globalization and industrialization. With an increasing need to reduce the system waste impact on the environment and an ever increasing global demand for energy, especially in developing countries, it is becoming extremely important to develop even more accurate and systematic approaches for improving the design of energy systems. Industry, transportation and buildings are the three main economic sectors that consume a significant amount of energy [1].

There are two characteristics that distinguish electricity from other commodities:

1. Electricity is not storable to a large extent, and,

2. Transport of electricity is linked to transmission networks.

These facts have consequences for the market structure, price behavior and products.

On the other hand, electricity demand depends on many factors such as temperature, humidity, precipitation, wind speed, cloud cover and amount of light.

The impact of temperature on both electricity demand and price has been studied in many papers. Results in Ref. [2] confirm that consumer responses to higher electricity prices are conditional on temperature levels, particularly during the daytime and for households with high overall levels of electricity consumption and previous experience of time-of-use tariffs. The relationship between electricity load and air temperature has an important dynamic component, and ignoring this appears to bias the estimated effects of temperature on load [3]. Research [4] estimates a forecasting equation for the hourly peak electricity demand for one day in the future. Everyday activities are also very important. There are different electricity demands and consumptions during weekends and working days, in winter and summer, holidays, etc.

A variety of methods and ideas have been tried for electricity price forecasting (EPF), with varying degrees of success. The Autoregressive Model (AR) is supposed to be a stochastic process that is composed of a weighted sum of the previous value and a white noise error.

Ref. [5] describes a price forecasting module based on neural networks and performance evaluation. An overview of modeling approaches that concentrates on practical applications of statistical methods for day-ahead forecasting, discusses interval forecasts, and moves on to quantitative stochastic models for derivatives pricing (jump-diffusion models and Markov regime-switching) is presented in work [6]. In [7] Zareipour begins by reviewing linear time series models and nonlinear models (regression splines, neural networks), and then uses them for forecasting hourly prices in the Ontario power market.

In this paper we propose a new model for the dynamics of temperature which forms the basis for pricing weather derivatives. The model generalizes the continuous-time autoregressive models suggested in Benth et al. [8], to allow for stochastic volatility effects. Due to transmission networks the electricity market is not global. Adjacent national electricity markets start to link to one another but the capacities give natural constraints. Therefore, we focus on the presentation of the Serbian electricity market which is relevant for our modeling.

This paper presents the electricity price forecasting for the tobacco production industry where averaged different values for a one-year period are given [9]. Electricity in the factory is mainly required for the propulsion of electric motors used for machine operation, compressed air production, pumps and fans. The total installed power of the electric motor is $1.2 \mathrm{MW}$. 


\section{PHASES OF FORECAST AND SIMULATION}

Probabilistic forecasting of electricity demand is an important task for all active market participants. For electricity consumption, $C_{t}$ at time $t$, fundamental model equation, given in Ref. [10], has the following form

$$
\log C_{t}=D_{t}+u\left(G_{t}\right)+R_{t},
$$

where $D_{t}$ is the deterministic seasonal pattern, $u\left(G_{t}\right)$ is the function of residual grid load $G_{t}$.

The regression method was utilized to remove the effects of trend and seasonality on hourly electricity load demands with the help of dummy variables, which presents weather conditions for the adequate region.

In modeling the seasonal cycle deterministically, there are several approaches. The discrete Fourier transform (DTF) is considered to be the most accurate since it removes the seasonal cycle both in the mean and in the variance. However, other researchers have proposed a novel approach in modeling the seasonal cycle, which is an extension of the DFT approach. Since small misspecification in a dynamic model can lead to large pricing errors, we incorporate a wavelet analysis in the modeling process in order to calibrate our model. The fundamental idea behind the wavelets is an analysis according to scale. The wavelet analysis is an extension of the Fourier transform, which superposes sins and cosines to represent other functions. The wavelet analysis decomposes a general function or signal into a series of (orthogonal) basis functions, called wavelets, with different frequency and time locations.

Seasonal mean function $D_{t}$ is assumed to be a real-valued continuous function. To capture the seasonal variations due to cold and warm periods of the year, and a possible increase in temperatures due to global warming and urbanization, a possible structure of $D_{t}$ could be

$$
D_{t}=a_{0}+b t+a+\sum_{i=1}^{I} a_{i} \sin \left(\frac{2 i \pi\left(t-f_{i}\right)}{365.25 \cdot 24}\right)+\sum_{l=1}^{J} b_{j} \cos \left(\frac{2 j \pi\left(t-g_{j}\right)}{365.25 \cdot 24}\right) .
$$

Parameter $a$ is the coefficient of the linear trend while $b$ is the slope. The estimated parameters of seasonal part $D_{t}$ are obtained using the nlinfit function in MATLAB. Amplitude $a$ indicates the difference between the daily winter and the daily summer temperature. For example, it is around $-4.4^{\circ} \mathrm{C}$ in London and $9.5^{\circ} \mathrm{C}$ in Chicago [11].

Now the wavelet analysis is used to identify the seasonal part. The results of the conducted wavelet analysis [12] indicate that the seasonal part of the temperature takes the following form

$$
\begin{aligned}
D_{t} & =a_{0}+b t+a+b_{1} \sin \left(\frac{2 \pi\left(t-f_{1}\right)}{365.25 \cdot 24}\right)+b_{2} \sin \left(\frac{2 \pi\left(t-f_{2}\right)}{365.25 \cdot 24}\right)+b_{3} \sin \left(\frac{2 \pi\left(t-f_{3}\right)}{365.25 \cdot 24}\right)+ \\
& +b_{4} \sin \left(\frac{2 \pi\left(t-f_{4}\right)}{365.25 \cdot 24}\right)+b_{5} \sin \left(\frac{2 \pi\left(t-f_{5}\right)}{365.25 \cdot 24}\right)+b_{6} \sin \left(\frac{2 \pi\left(t-f_{6}\right)}{365.25 \cdot 24}\right) .
\end{aligned}
$$


Depending on the geographical area, the authors indicate different values of coefficients $a$ and $b$. For example, for the New York these coefficients are presented in Ref. [13].

Parameter $a_{0}$ is the amplitude of the sinusoid of the seasonal variance and $f_{1^{-}} f_{6}$ is the angle that refers to the maximum and minimum of the temperature in the year. All parameters are statistically significant.

Parameter $G_{t}$ in Eq. (1) denotes the residual grid load, i.e. the total system load minus its deterministic part where function $u\left(G_{t}\right)$ could be any suitable function; a linear approach is found sufficient [10].

Residual time series $R_{t}$ in Eq. (1) is often modeled by a Gaussian white noise process. The time simulation of the white noise process is given in Fig. 1.

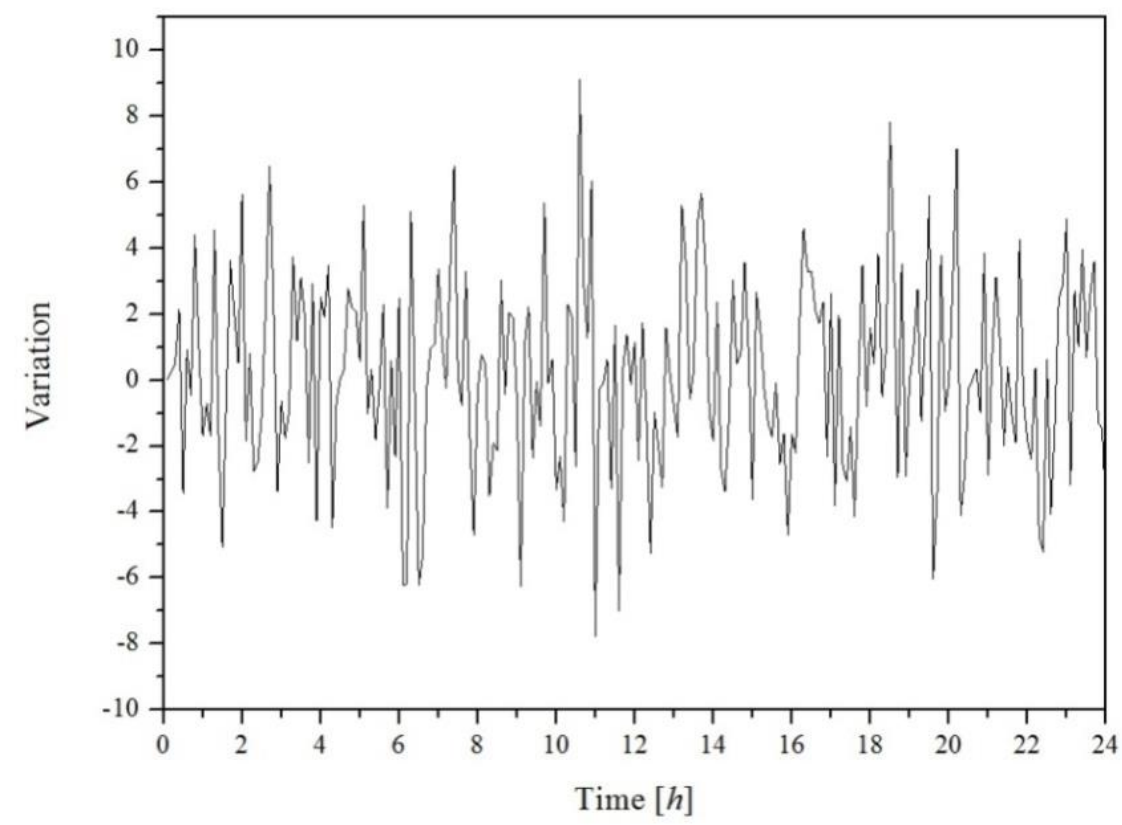

Fig. 1 Simulation of white noise process

On the basis of Eqs. (1) and (3), the simulation scheme was made in MATLAB software and used for determining and predicting the costs for the different values of parameters from Eq. (3). This scheme is initially developed in [14] and it is presented in Fig. 2. 


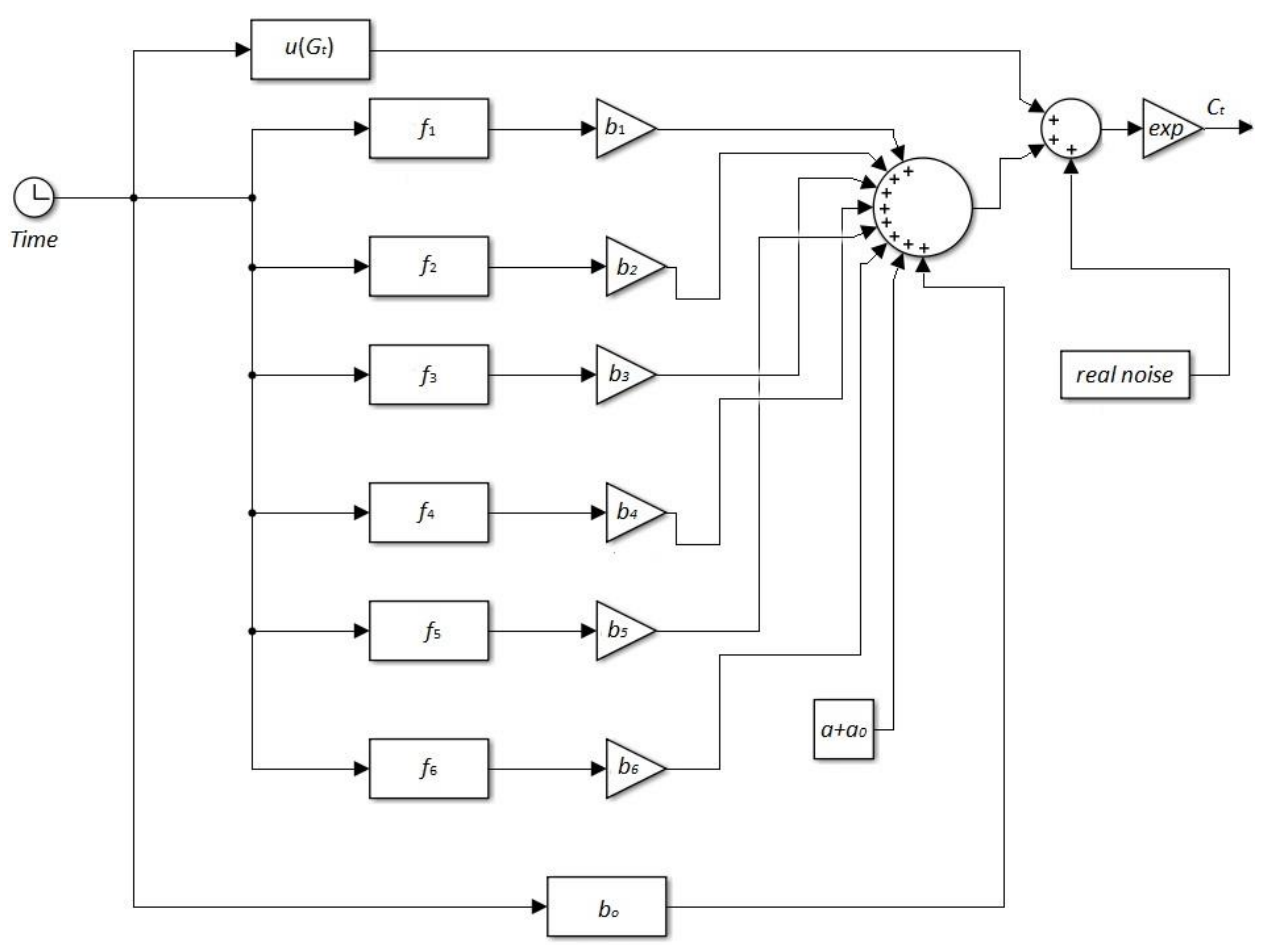

Fig. 2 Simulation scheme

\section{NUMERICAL RESULTS}

To determine the optimal values of electricity consumption the numerical Monte Carlo method was applied. This method finds its application in almost every field of study and presents a broad class of computational algorithms that rely on repeated random sampling to obtain numerical results. The Monte Carlo method can be used to predict the future economic parameters which affect the operation cost of some industry production [15]. This simulation uses time step size of $\Delta t=0.1 \mathrm{~s}$, while the number of simulations is $N=2000$.

As the example, the numerical results were performed for a tobacco production where firstly, according to the average monthly energy consumption (Table 1 ), function $u\left(G_{t}\right)$ is determined and presented in Fig. 3. The tobacco factory described in [9] is supplied with electricity through four transformer stations with the total leased power of $1.5 \mathrm{MW}$. The consumption of electricity in the factory per month according to the data for the year 2006 is given in Table 1. 
Table 1 The electricity consumption is given by months for the year 2006 [9]

\begin{tabular}{lc}
\hline 2006 & $\begin{array}{c}\text { Consumed } \\
\text { electricity } \\
(\mathrm{kWh})\end{array}$ \\
\hline January & 762000 \\
February & 694500 \\
March & 649500 \\
April & 504000 \\
May & 732000 \\
June & 931500 \\
July & 793500 \\
August & 655500 \\
September & 805500 \\
October & 792000 \\
November & 783000 \\
December & 744000 \\
\hline In total & 8847000 \\
\hline
\end{tabular}

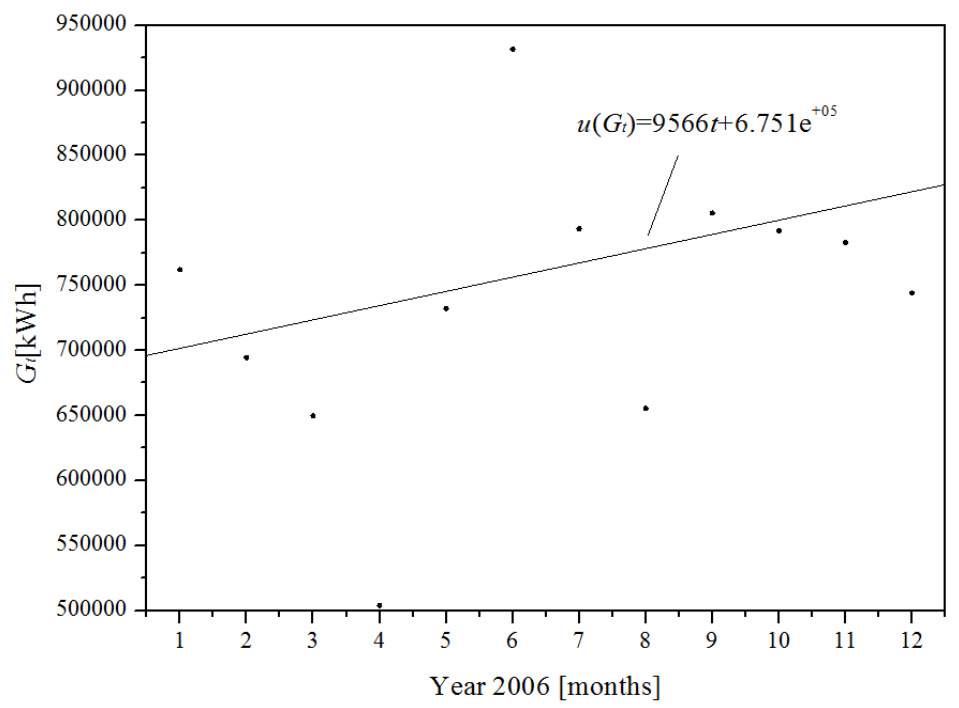

Fig. 3 Approximated linear function $u\left(G_{t}\right)$ according to values given in Table 1

Many companies have an electricity demand that is, except for seasonality and some noise, quite homogeneous. They have fixed opening hours during which the electricity demand reaches a certain level, whereas at other hours the consumption drops to what we refer to as the standby level. There is a very homogeneous structure during the weekdays and the low demand on Sundays. There are also special events and holidays. However, these different regimes during opening hours and closing hours are easy to predict. Therefore, we can de-seasonalize the data to get something quite homogeneous. Now, according to average 
time conditions in most cities in Europe that correspond to weather conditions in Serbia, the parameters which are used in Eq. (3) are presented in Table 2. These parameters are presented for working days, weekends, summer, spring, winter and autumn.

Table 2 The estimated parameters

\begin{tabular}{ll}
\hline \multicolumn{2}{c}{$a_{0}=-0.0008 ; a=1.242$} \\
\hline$b_{1}=7.6994$ & $f_{1}=-73.2644$ \\
$b_{2}=0.1317$ & $f_{2}=95.0642$ \\
$b_{3}=0.0469$ & $f_{3}=-640.2319$ \\
$b_{4}=-0.2743$ & $f_{1}=183.109$ \\
$b_{5}=-0.3445$ & $f_{1}=-13.1151$ \\
$b_{6}=0.0796$ & $f_{1}=-134.5803$ \\
\hline
\end{tabular}

According to the parameters from Table 2, the determination and prediction of energy costs are given in Fig. 4. Initial time in this figure shows the energy consumption estimated for a previous month where the further costs prediction are given for the next ten days.

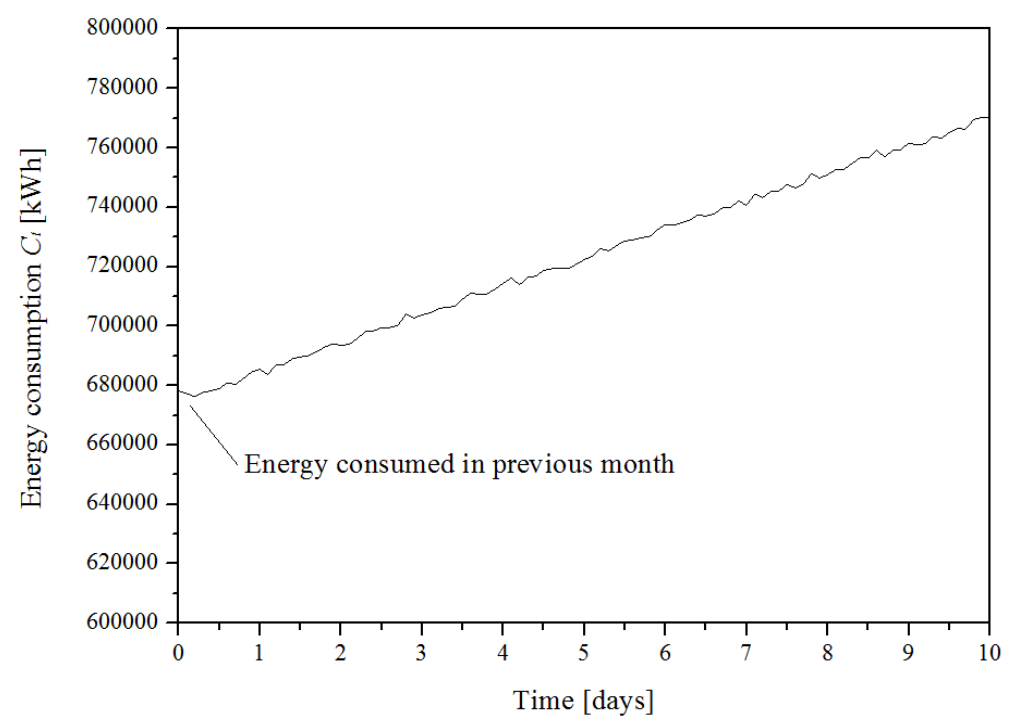

Fig. 4 Approximated energy consumption for parameters given in Table 2

These results are also given for larger variations in an energy system which is taken into account using the white noise process $R_{t}$ in Eq. (1). The results are presented in Fig. 5. 


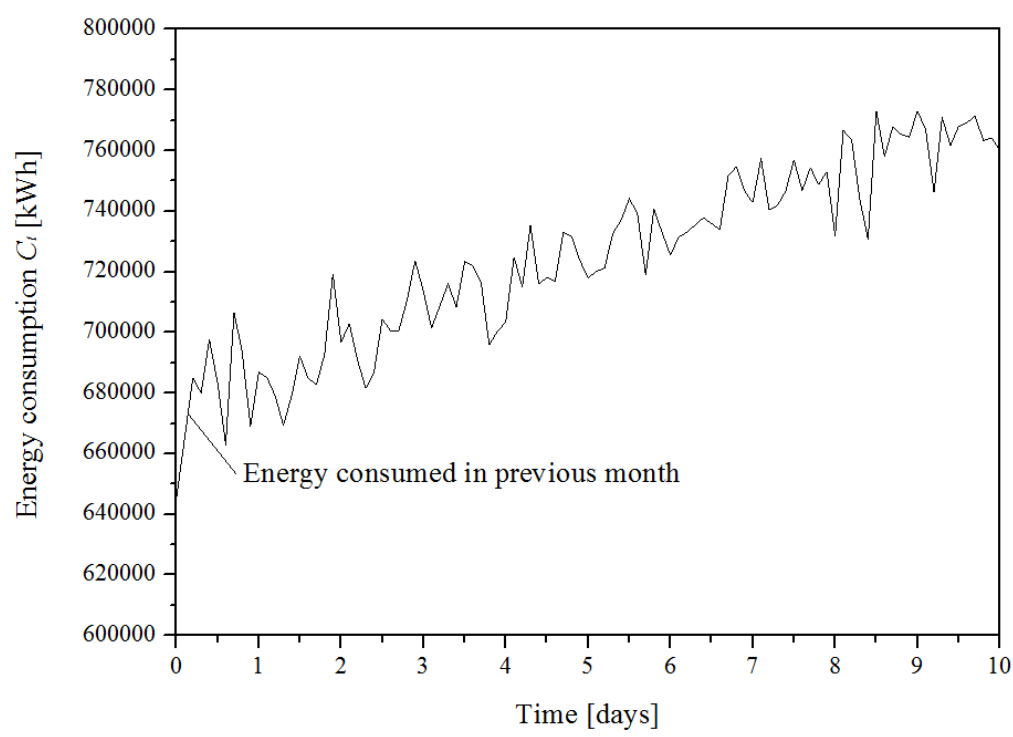

Fig. 5 Approximated energy consumption for larger variations in energy system

\section{CONCLUSION}

This paper introduced a time-series model for forecasting the electricity consumption of a tobacco production company. The Monte Carlo model was suggested for probabilistic forecasting of electricity load of an industrial company. The time-series method forecasts energy demand by the patterns and trends found in the data. When using a time-series method, the researcher uses statistical extrapolation of loads based upon historical data.

Electricity is one of the most important and exploited forms of energy, and it is widely used for different kinds of needs. Electricity consumption varies according to the season and the time of day. Energy consumption varies from season to season because more electricity is used during the winter and summer months when it is hotter or colder than in the spring and fall months when the temperatures are usually moderate. Nowadays electricity is essential for economic development especially for the industrial sector. Decision makers around the world widely use energy demand forecasting as one of the most important policy tools. One of the decision makers' dilemmas is how to forecast electricity demands.

The main innovation of this paper is the Monte Carlo model which is used to forecast electricity demands. The Monte Carlo simulation produces not only one answer, but rather a series of answers or a range over which the results vary as a function of probability of occurrence and also a most expected result. In other words, the Monte Carlo simulation generates hundreds of alternative (scenarios) for a project. The answer may fall anywhere within the range of the results produced.

This paper described the electricity demand for tobacco production in a company by dummy calendar variables. The production regime process is modeled as a seasonal pattern combined with an autoregressive process, while in the standby regime we assume the demand to follow a simple white noise process. The Autoregressive Model (AR) is supposed 
to be a stochastic process which is composed of a weighted sum of the previous value and a white noise error. The impact of weather conditions on both electricity demand and price has been considered through the seasonal pattern.

\section{REFERENCES}

1. Khosravani, H., Castilla M., Manuel Berenguel M., Ruano A., Ferreira P., 2016, A comparison of energy consumption prediction models based on neural networks of a bioclimatic building, Energies, 9(57), pp.1-24.

2. Henley, A., Peirson, J., 1994, Residential energy demand and the interaction of price and temperature: British experimental evidence, Energy Economics, 20, pp.157-171.

3. Peirson, J., Henley, A., 1994, Electricity load and temperature issues in dynamic specification, Energy Economics, 16, pp. 235-243.

4. Engle, R.F., Mustafa, C., Rice, J., 1992, Modelling peak electricity demand, Journal of Forecasting, 11, pp. 241-251.

5. Shahidehpour, M., Yamin, H., Li, Z., 2002, Market operations in electric power systems: forecasting, scheduling, and risk management, John Wiley and Sons Ltd, New York, United States.

6. Weron, R., 2006, Modeling and forecasting electricity loads and prices: a statistical approach, John Wiley and Sons Ltd, Chichester, United Kingdom.

7. Zareipour, H., 2008, Price-based energy management in competitive electricity markets, VDM Verlag Dr. Mueller e.K., Germany.

8. Benth, F.E., Saltyte-Benth, J., Koekebakker, S., 2007, Putting a price on temperature, Scandinavian Journal of Statistics, 34,(4), pp. 746-767.

9. Ilić, G., 2007, Preliminary energy review factory-tobacco industry "Vranje", Annual Report, Faculty of Mechanical Engineering, University of Niš, Serbia.

10. Berk, K., Müller, A., 2016, Probabilistic forecasting of medium-term electricity demand: a comparison of time series models, Journal of Energy Markets, 9(2), pp. 1-20.

11. Zapranis, A., Alexandridis, A., 2011, Modeling and forecasting cumulative average temperature and heating degree day indices for weather derivative pricing, Neural Computing and Applications, 20(6), pp. 787-801.

12. Benth, F.E., Saltyte-Benth, J., 2007, The volatility of temperature and pricing of weather derivatives quantitative finance, Quantitative Finance 7(5), pp. 553-561

13. Göncü, A., Liu, Y., Ökten, G., Yousuff Hussaini, M., 2016, Uncertainty and robustness in weather derivative models, Monte Carlo and Quasi-Monte Carlo Methods, Springer Proceedings in Mathematics \& Statistics, 163, Springer, Cham.

14. Laković M., Pavlović I., Banjac M., Jović M., 2017, Determination and prediction of electricity consumption using the Monte Carlo simulation method, SIMTERM, Soko Banja, Serbia, ISBN 978-866055-098-1.

15. Laković, M., Pavlović, I., Jović, M., 2017, Aplication of Monte Carlo method for large steam condenser performances determination in variable operating conditions, KODIP, Budva, Montenegro, pp. 251-258. 